\title{
INTOXICAÇÕES EXÓGENAS EM CRIANÇAS MENORES DE SEIS ANOS ATENDIDAS EM HOSPITAIS DA REGIÃO METROPOLITANA DO RIO
}

\section{DE JANEIRO}

\section{Guilherme Loureiro Werneck*1, Maria Helena Hasselmann ${ }^{2}$}

Trabalho elaborado no Instituto de Medicina Social, Universidade do Estado do Rio de Janeiro Instituto de Estudos em Saúde Coletiva, Universidade Federal do Rio de Janeiro e Instituto de Nutrição, Universidade do Estado do Rio de Janeiro, RJ

\author{
*Correspondência: \\ Instituto de Medicina Social \\ - Universidade do Estado \\ do Rio de Janeiro \\ Rua São Francisco Xavier \\ 524 - 70 andar - Bloco D \\ CEP 20559-900 \\ Rio de Janeiro - RJ \\ Tel: 21-2587-7303 \\ Fax: -21-2264-1142 \\ gwerneck@ims.uerj.br
}

\begin{abstract}
RESUMO
OвJEtivo. Descrever o perfil dos casos de intoxicações exógenas de crianças admitidas em hospitais de emergência da região metropolitana do Rio de Janeiro, durante três anos.

Métodos. Realizou-se levantamento dos dados a partir dos boletins de atendimento de emergência, tendo sido identificados os casos suspeitos ou confirmados de intoxicação nas seguintes categorias: drogas, medicamentos e substâncias biológicas; solventes orgânicos e hidrocarbonetos halogenados; produtos químicos; monóxido de carbono e outros gases, fumaças e vapores; e pesticidas.

Resultados. Foram registrados 1.574 casos de intoxicação entre crianças até cinco anos de idade, sendo que cerca de $40 \%$ dos casos envolveu produtos químicos de uso doméstico, $35 \%$ os medicamentos, e $15 \%$ algum tipo de pesticida. Mais da metade das intoxicações por pesticidas envolveu o chumbinho, um produto ilegalmente vendido como raticida, que frequentemente contém o agrotóxico carbamato. A distribuição dos agentes envolvidos variou significativamente segundo sexo, sendo a frequência relativa das intoxicações por medicamentos e chumbinho maior no sexo feminino em comparação ao masculino. Observou-se que a participação dos produtos químicos de uso doméstico decresceu com a idade, enquanto aumentou a dos medicamentos como agentes da intoxicação. No período analisado não observou-se redução significativa no número total de casos registrados anualmente.

Conclusão. Fortalecer a rede de Centros de Controle de Intoxicações, intervir na linha de produção de embalagens para medicamentos e produtos químicos, aumentar a fiscalização sobre a comercialização ilegal do chumbinho, e enfatizar atividades de educação em saúde são ações que podem contribuir para mudar este panorama.
\end{abstract}

Unitermos: Toxicologia. Envenenamento. Praguicidas, Medicamentos. Acidentes. Pediatria.

\section{INTRODUÇÃO}

As intoxicações exógenas na infância representam um importante problema de saúde pública mundial. Em 2002, segundo estimativas da Organização Mundial da Saúde, cerca de 350 mil mortes devido às intoxicações ocorreram no mundo, sendo pelo menos 10\% em menores de 15 anos de idade. ${ }^{1}$ Em 1997, inquérito de base populacional nos Estados Unidos estimou em cerca de 550 mil o número de episódios de intoxicações em menores de seis anos de idade que necessitaram de atenção médica nos três meses anteriores à entrevista. ${ }^{2}$

Crianças menores de cinco anos de idade formam um grupo particularmente vulnerável às intoxicações acidentais, principalmente devido à curiosidade inerente à idade, elas exploram o ambiente de forma íntima com todos os sentidos, o que favorece o contato e a ingestão de agentes tóxicos. ${ }^{3} \mathrm{Na}$ maior parte das vezes a intoxicação ocorre no próprio local de moradia sendo que as substâncias envolvidas não estão adequadamente armazenadas. ${ }^{4-7} \mathrm{~A}$ administração errônea de substâncias tóxicas por um adulto é outra circunstância comumente associada à intoxicação infantil, principalmente em menores de um ano de idade. ${ }^{4}$

A Associação Americana de Centros de Controle de Intoxicação (CCI) registrou, em 2004, mais de 2,4 milhões de intoxicações, destas cerca de metade ocorreu em crianças até cinco anos de idade com discreto predomínio no sexo masculino. ${ }^{8}$ Mais de $98 \%$ das intoxicações foram consideradas não intencionais e as principais substâncias envolvidas foram os cosméticos e produtos de higiene pessoal $(13,4 \%)$, produtos de limpeza (10\%), analgésicos $(7,9 \%)$, produtos tópicos $(7,4 \%)$, corpos estranhos $(7,3 \%)$, preparações para resfriados e tosse $(5,4 \%)$, plantas $(4,4 \%)$, pesticidas $(4,2 \%)$, vitaminas $(3,9 \%)$, antihistamínicos $(2,8 \%)$, e antimicrobianos $(2,7 \%){ }^{8}$ 
Em 2003, no Brasil, o Sistema Nacional de Informações Tóxico-Farmacológicas por meio da rede de Centros de Informação e Assistência Toxicológica registrou 82.716 casos de intoxicação humana e 530 óbitos. ${ }^{9}$ Assim como no caso norteamericano, a distribuição dos casos de intoxicação não variou segundo sexo e cerca de $25 \%$ dos casos ocorrerram abaixo dos cinco anos de idade. Os principais agentes tóxicos envolvidos nas intoxicações de menores de cinco anos foram os medicamentos $(38,3 \%)$, domissanitários $(18 \%)$, produtos químicos industriais $(8,7 \%)$, agrotóxicos $(7,1 \%)$, mordeduras e picadas por animais peçonhentos $(6,7 \%)$, raticidas $(5,6 \%)$, plantas $(4,1 \%)$, mordeduras e picadas por animais não peçonhentos (5,8\%), e cosméticos $(2,7 \%){ }^{9}$

Em 2003, outro estudo sobre as intoxicações em menores de cinco anos de idade registradas no Centro de Informação Toxicológica do Rio Grande do Sul, mostrou que as intoxicações eram menos frequentes em menores de um ano e predominavam discretamente no sexo masculino (53\%). Em relação às circunstâncias de ocorrência, identificou-se a via oral como principal meio de exposição (88\%), a localização preferencial do agente tóxico no chão da sala de estar ou do quarto (67\%), os medicamentos, especialmente os analgésicos, como principais agentes tóxicos (42\%), seguidos dos domissanitários (16\%). ${ }^{6}$

É importante salientar que, apesar de se constituir em um sistema de informações extremamente valioso para o conhecimento da magnitude das intoxicações, os dados oriundos dos $\mathrm{CCls}$ são possivelmente subestimados, na medida em que a notificação de casos de intoxicação não é compulsória, mas decorre essencialmente da necessidade da equipe de saúde ou familiares em obter informações sobre diagnóstico e tratamento. $\mathrm{Na}$ medida em que as equipes dos serviços de emergência se tornam proficientes nestas atividades elas podem não sentir a necessidade de recorrer aos CCls, implicando em possível subnotificação expressiva. Os dados norte-americanos sugerem que cerca de $25 \%$ dos casos de intoxicação em menores de seis anos de idade que necessitam de assistência médica não chegam a ser notificados aos CCls. ${ }^{2}$ Mais grave, entretanto, é o potencial de subnotificação seletiva, principalmente em função da gravidade da lesão, contribuindo para distorcer o perfil de substâncias envolvidas.

Os serviços de emergência são uma fonte alternativa de dados sobre intoxicações, mas apresentam também uma série de limitações. Por um lado, não está disponível uma base de dados especificamente construída para registrar os motivos deste tipo de atendimento, por outro, as informações incluídas nos boletins de atendimento são, em geral, de má-qualidade e insuficientes para caracterização completa do evento. Ainda assim, um perfil das intoxicações exógenas obtido a partir desta fonte de dados poderia contribuir para uma descrição mais abrangente da magnitude das intoxicações da infância. Admitindo que na infância estes eventos tendem a ser potencialmente graves, supõem-se que a maioria deles precise de atenção de saúde em um serviço de emergência e que, nestes casos, os boletins de registro de atendimento tendam a ser mais adequadamente preenchidos. Nessa perspectiva, o objetivo deste trabalho é caracterizar o perfil das intoxicações na infância a partir de dados obtidos em oito hospitais de emergência localizados na região metropolitana do Rio de Janeiro, durante o período de três anos.

\section{MÉTOdos}

\section{Tipo e locais de estudo}

Trata-se de uma série de estudos seccionais mensais realizados por meio de levantamento retrospectivo de dados com base nos boletins de atendimento de emergência de oito hospitais localizados na região metropolitana do Rio de Janeiro (Hospital Estadual Getúlio Vargas, Hospital Estadual Rocha Faria, Hospital Estadual Adão Pereira Nunes, Hospital Estadual Pedro II, Hospital Municipal Miguel Couto, Hospital Municipal Salgado Filho, Hospital Municipal Lourenço Jorge, Hospital Municipal Souza Aguiar), cobrindo um período de 36 meses.

\section{População de estudo}

A população de estudo compreende os casos de intoxicação exógena admitidos nos serviços de emergência dos hospitais selecionados. Optou-se por obter uma amostragem de conveniência, baseada na seleção aleatória de 17 dias de atendimento a cada mês de estudo e em cada hospital. Todos os registros de atendimento no serviço de emergência destes dias foram examinados, perfazendo mais da metade dos atendimentos de emergência em cada hospital.

\section{Procedimentos}

Ficha de coleta de dados: Optou-se por usar o mesmo formulário utilizado para registro de atendimentos de emergência (boletim de emergência), de forma que toda a informação registrada fosse copiada ipsi litere para esta ficha, a ser então revista, codificada e digitada pela coordenação da pesquisa. Por razões éticas esta ficha continha um carimbo de "SEM EFEITO" e o nome do paciente não era identificado. Ressalta-se que todos os procedimentos utilizados nesta investigação foram aprovados pelo Comitê de Ética em Pesquisa da UFRJ.

Recrutamento e treinamento da equipe de coleta de dados: A equipe (coordenadora de atividade de campo e auxiliares de pesquisa) foi recrutada em Abril de 2002 e submetida a sessões de treinamento iniciadas neste mesmo mês, seguidas de reuniões bimestrais de atualização.

Supervisão das atividades de campo: A coordenação de atividades de campo realizou supervisão contínua nos hospitais de forma a garantir o bom andamento do trabalho. Esta atividade incluiu revisão de todos boletins de emergência de uma amostra de dias de atendimento avaliados pelos auxiliares de pesquisa de forma a identificar problemas e erros. Em todos os hospitais incluídos no estudo, tal trabalho foi realizado mensalmente em pelo menos $10 \%$ dos dias de atendimento. O coordenador esteve em contato com os auxiliares de pesquisa semanalmente, e eventualmente agendou encontros diretamente nos hospitais para avaliar o desempenho e qualidade do trabalho realizado.

Reuniões com os auxiliares de pesquisa: O treinamento e a supervisão foram reforçados e atualizados bimestralmente em reuniões entre o coordenador geral da pesquisa, a coordenadora de atividades de campo e os auxiliares de pesquisa. Nestes encontros, foram utilizados exemplos de boletins de ocorrência com diferentes histórias clínicas, algumas dúbias, outras típicas, para avaliar a capacidade dos auxiliares de pesquisa de discriminar estas diferentes situações. Em algumas ocasiões houve a necessidade de reuniões individualizadas com 
alguns auxiliares de pesquisa para esclarecer dúvidas e reforçar condutas padronizadas.

Coleta de dados: 0 período de cobertura do trabalho foi de abril de 2001 a março de 2004 . Foram coletados todos os casos suspeitos ou confirmados de intoxicação que se enquadrassem nas seguintes categorias:

- Drogas, medicamentos e substâncias biológicas;

- Solventes orgânicos e hidrocarbonetos halogenados e vapores;

- Produtos químicos (corrosivos, fertilizantes, sabões e detergentes, colas e adesivos, metais, plantas venenosas e tintas e corantes)

- Monóxido de carbono e outros gases, fumaças e vapores;

- Pesticidas

Além das intoxicações por alucinógenos, foram também excluídas as intoxicações por álcool, as de origem alimentar e as lesões provocadas por animais peçonhentos ou não.

\section{Processamento e análise de dados}

Os dados foram preliminarmente armazenados em planilha eletrônica. Para fins de análise, os agentes da intoxicação foram subdivididos em cinco grupos:

- Chumbinho (produto ilegalmente vendido como raticida, que freqüentemente contém em sua composição o agrotóxico carbamato);

- Medicamentos (drogas, medicamentos e substâncias biológicas);

- Produtos químicos de uso doméstico (domissanitários, cosméticos, adesivos e colas);

- Outros pesticidas;

- Outros agentes.

Os seguintes critérios foram utilizados para definição da intoxicação por chumbinho:

Caso confirmado: caso alegado de intoxicação por chumbinho com

- Saída de grânulos compatíveis na lavagem gástrica ou,

- Presença de miose associada à sialorreia e/ou miofasciculações e tratamento com atropina.

Caso muito provável: caso alegado de intoxicação por chum-

binho com

- Presença de pelo menos um dos seguintes sintomas: miose, sialorreia ou miofasciculações e tratamento com atropina.

Caso provável: caso alegado de intoxicação por chumbinho com

- Tratamento com atropina ou

- Presença de pelo menos um dos seguintes sintomas: miose, sialorreia ou miofasciculações.

Caso possível: caso alegado de intoxicação por carbamato

ou por "veneno de rato" com

- Tratamento com atropina ou

- Presença de pelo menos um dos seguintes sintomas: miose, sialorreia ou miofasciculações.

Caso de contato com chumbinho sem intoxicação: caso alegado de intoxicação por chumbinho sem miose, sialorreia ou miofasciculações e sem tratamento com atropina.

Posteriormente, os dados foram armazenados em banco de dados elaborado especificamente para este fim em EPIINFO e analisados utilizando-se o aplicativo para análise estatística
Figura 1 - Distribuição proporcional dos casos de intoxicação infantis segundo categorias dos agentes envolvidos, Rio de Janeiro

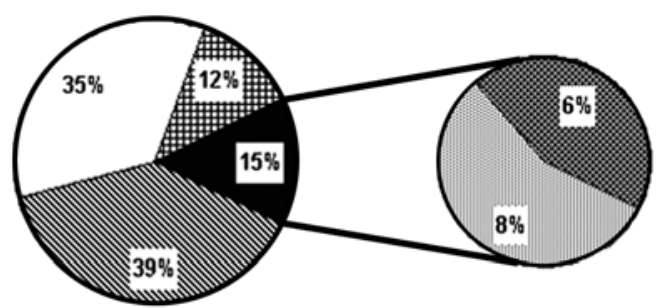

ه Produtos químicos de uso doméstico $\square$ Medicamentos

田 Outros

Chumbinho

O Outros Pesticidas

Stata 9.2. Utilizou-se o teste de Qui quadrado $\left(x^{2}\right)$ para avaliar as diferenças na distribuição dos agentes de intoxicação segundo sexo, faixa etária (0-1, 2-3 e 4-5 anos de idade) e período do estudo (12 meses iniciais, 12 meses intermediários e 12 meses finais da pesquisa). Utilizou-se também o teste não paramétrico de tendência linear de Cuzick para avaliar se houve mudanças significativas na frequência das intoxicações durante os três períodos analisados. Considerou-se um valor de p de 5\% como critério para significância estatística dos resultados.

\section{Resultados}

Nestes oito hospitais foram identificados 1.574 casos suspeitos ou confirmados de intoxicação entre crianças até cinco anos de idade. A Figura 1 mostra a distribuição proporcional dos casos de intoxicação segundo o tipo de agente envolvido. Observa-se que 39\% dos casos envolveram produtos químicos de uso doméstico, $35 \%$ os medicamentos e $15 \%$ algum tipo de pesticida. Mais da metade das intoxicações por pesticidas envolveu o chumbinho, quando se considera o critério mais amplo para definir este tipo de intoxicação (todas as categorias de confirmação). Se o critério para diagnóstico de intoxicação pelo chumbinho se restringe apenas aos casos confirmados, muito prováveis e prováveis, então esta proporção cai para cerca de $25 \%$.

A Tabela 1 mostra distribuição dos agentes segundo sexo. Há diferença significativa no perfil de agentes de intoxicação, sendo relativamente mais frequentes as intoxicações por chumbinho e medicamentos no sexo feminino.

A Tabela 2 mostra a distribuição dos casos de intoxicação segundo o tipo de agente envolvido e faixa etária. Quase $85 \%$ das intoxicações ocorreram até três anos de idade, sendo que a participação dos produtos químicos de uso doméstico decresceu com a idade, enquanto aumentou a dos medicamentos como agentes da intoxicação.

A Tabela 3 mostra a evolução das intoxicações nos três períodos do estudo. Ainda que não tenha sido possível identificar uma tendência linear decrescente estatisticamente significante (valor de $p=0,53$ ), observou-se uma redução de $8 \%$ no número total de casos anuais, fundamentalmente devido à redução em 
Tabela 1 - Distribuição dos casos de intoxicação segundo o tipo de agente envolvido e sexo

\begin{tabular}{|c|c|c|c|c|c|c|}
\hline \multirow[b]{2}{*}{ Agente } & \multicolumn{2}{|c|}{ Feminino } & \multicolumn{2}{|c|}{ Masculino } & \multicolumn{2}{|c|}{ Total } \\
\hline & $\mathrm{N}$ & $\%$ & $\mathrm{~N}$ & $\%$ & $\mathrm{~N}$ & $\%$ \\
\hline Chumbinho & 62 & 9,6 & 51 & 6,8 & 113 & 8,1 \\
\hline Medicamentos & 245 & 38 & 246 & 32,6 & 491 & 35,1 \\
\hline Produtos químicos de uso doméstico & 233 & 36,2 & 299 & 39,7 & 532 & 38,1 \\
\hline Outros pesticidas & 42 & 6,5 & 50 & 6,6 & 92 & 6,6 \\
\hline Outros agentes & 62 & 9,6 & 108 & 14,3 & 170 & 12,2 \\
\hline Total & 644 & 100 & 754 & 100 & 1398 & 100 \\
\hline
\end{tabular}

$X^{2}=13,83$ e valor de $p=0,008$

Tabela 2 - Distribuição dos casos de intoxicação segundo o tipo de agente envolvido e faixa etária

\begin{tabular}{|c|c|c|c|c|c|c|c|c|}
\hline \multirow[b]{2}{*}{ Agente } & \multicolumn{2}{|c|}{$0-1$ anos } & \multicolumn{2}{|c|}{$2-3$ anos } & \multicolumn{2}{|c|}{ 4-5 anos } & \multicolumn{2}{|c|}{ Total } \\
\hline & $\mathrm{N}$ & $\%$ & $\mathrm{~N}$ & $\%$ & $\mathrm{~N}$ & $\%$ & $\mathrm{~N}$ & $\%$ \\
\hline Chumbinho & 54 & 9,8 & 42 & 6,2 & 25 & 10 & 121 & 8,2 \\
\hline Medicamentos & 145 & 26,2 & 258 & 38,2 & 116 & 46,2 & 519 & 35,1 \\
\hline Produtos químicos de uso doméstico & 249 & 45 & 252 & 37,3 & 70 & 27,9 & 571 & 38,6 \\
\hline Outros pesticidas & 44 & 8,0 & 37 & 5,5 & 13 & 5,2 & 94 & 6,4 \\
\hline Outros agentes & 61 & 11 & 87 & 12,9 & 27 & 10,8 & 175 & 11,8 \\
\hline Total & 553 & 100 & 676 & 100 & 251 & 100 & 1480 & 100 \\
\hline
\end{tabular}

$X^{2}=26,33$ e valor de $p<0,001$ para a comparação entre 0-1 e 2-3 anos

$X^{2}=35,94$ e valor de $p<0,001$ para a comparação entre $0-1$ e 4-5 anos

$X^{2}=11,84$ e valor de $p=0,019$ para a comparação entre 2-3 e 4-5 anos

Tabela 3 - Evolução dos casos de intoxicação segundo o tipo de agente envolvido e período

\begin{tabular}{|c|c|c|c|c|c|c|c|c|}
\hline \multirow[b]{2}{*}{ Agente } & \multicolumn{2}{|c|}{ Período $1^{*}$} & \multicolumn{2}{|c|}{ Período $2^{*}$} & \multicolumn{2}{|c|}{ Período $3^{*}$} & \multicolumn{2}{|c|}{ Total } \\
\hline & $\mathrm{N}$ & $\%$ & $\mathrm{~N}$ & $\%$ & $\mathrm{~N}$ & $\%$ & $\mathrm{~N}$ & $\%$ \\
\hline Chumbinho & 43 & 8,4 & 43 & 8,6 & 35 & 7,5 & 121 & 8,2 \\
\hline Medicamentos & 182 & 35,5 & 162 & 32,4 & 175 & 37,5 & 519 & 35,1 \\
\hline Produtos químicos de uso doméstico & 206 & 40,2 & 205 & 41 & 160 & 34,3 & 571 & 38,6 \\
\hline Outros pesticidas & 28 & 5,5 & 29 & 5,8 & 37 & 7,9 & 94 & 6,4 \\
\hline Outros agentes & 54 & 10,5 & 61 & 12,2 & 60 & 12,9 & 175 & 11,8 \\
\hline Total & 513 & 100 & 500 & 100 & 467 & 100 & 1480 & 100 \\
\hline
\end{tabular}

* Período 1: abril de 2001 a março de 2002; Período 2: abril de 2002 a março de 2003; e Período 3: abril de 2002 a março de 2004

$\mathrm{X}^{2}=1,44$ e valor de $\mathrm{p}=0,837$ para a comparação entre período 1 e período 2

$X^{2}=6,16$ e valor de $p=0,188$ para a comparação entre período 1 e período 3

$\mathrm{X}^{2}=6,73$ e valor de $\mathrm{p}=0,151$ para a comparação entre período 2 e período 3

24\% das intoxicações por produtos químicos de uso doméstico. Esta tendência diferenciada provoca uma mudança na distribuição proporcional dos agentes tóxicos. Os produtos químicos de uso doméstico eram os principais agentes no primeiro período de observação e são substituídos pelos medicamentos no período final do estudo.

\section{Discussão}

As intoxicações exógenas são importantes causas de morbidade infantil no cenário mundial, representando uma carga alta tanto em termos de morbidade como no que diz respeito aos custos de atenção hospitalar. Os resultados do presente estudo destacam a importância destes eventos também em nosso meio. Considerando que os dados foram obtidos de uma amostra de aproximadamente $56 \%$ dos dias de atendimento nos oito hospitais estudados durante os três anos de estudo, estima-se que o número total de intoxicações em crianças até cinco anos de idade nestes hospitais deva alcançar valores próximos a 1.200 eventos por ano. 0 problema deve ser ainda mais grave, já que os hospitais incluídos neste estudo são responsáveis por apenas uma parcela dos atendimentos de emergência realizados no Estado do Rio de Janeiro.

Os resultados deste estudo salientam algumas outras informações relevantes, particularmente em relação aos agentes envolvidos nas intoxicações. Destacam-se, primeiramente, os produtos químicos de uso doméstico e dos medicamentos, responsáveis por cerca de $75 \%$ das ocorrências, resultado comparável aos encontrados em outros estudos. ${ }^{6,7,10}$ Este achado 
WeRneck GL et al.

sustenta as recomendações para a adoção da Embalagem Especial de Proteção à Criança (EEPC) em medicamentos e produtos químicos de uso doméstico que apresentem potencial de risco à saúde como forma de prevenir intoxicações acidentais entre crianças, conforme salientados em outros trabalhos. ${ }^{4,5,11,12}$ Vale ressaltar que o projeto de lei 4841/1994 instituindo a EEPC no Brasil foi apresentado no Congresso Nacional em 1994, e ainda encontra-se em tramitação. ${ }^{12,13} \mathrm{Um}$ maior controle sobre a propaganda, a prescrição e a aquisição de medicamentos, assim como cuidados no armazenamento de produtos de uso doméstico, poderiam também contribuir para o controle das intoxicações, com repercussões potenciais na sua letalidade. .,11,12 $^{2}$

Importante também é a identificação de que quase 10\% das intoxicações ocorreram devido à ingestão do "chumbinho", produto comercializado de forma ilegal e propalado como um raticida eficiente. Na realidade, o "chumbinho" é um nome popular atribuído a um conjunto de produtos granulados com aparência acinzentada contendo produtos tóxicos, em geral, mas nem sempre, o agrotóxico carbamato. ${ }^{14} \mathrm{~A}$ diminuição da oferta do "chumbinho" por meio de uma maior fiscalização sobre sua comercialização e a apreensão do produto, associada à difusão de informações sobre o seu potencial letal e baixo poder rodenticida, poderia reduzir a magnitude e a gravidade destes eventos.

A participação de outros pesticidas que não o chumbinho (agrotóxicos de uso doméstico, na lavoura ou de uso veterinário) foi relativamente modesta, mas relevante considerando seu potencial letal. Em outras regiões do Brasil e do mundo o chumbinho não aprece como problema de saúde pública tão grave como no Rio de Janeiro, optou-se por separá-lo em nossa análise, mas esta decisão também dificulta a comparação com os resultados de outros estudos. Porém, considerando que o grupo dos "outros pesticidas" representaria aproximadamente o conjunto de agrotóxicos disponíveis em outras regiões, pode-se perceber que a prevalência de cerca de $7 \%$ obtida nesta investigação não destoa significativamente dos resultados de outros estudos $6,8,10,15$, ainda que um estudo na Turquia tenha encontrado cerca de $17 \%$ de intoxicações por pesticidas entre menores de cinco anos de idade atendidos em um serviço de emergência ${ }^{16}$.

Apesar de significantes, as diferenças no perfil de agentes envolvidos nas intoxicações segundo sexo não foram expressivas, o que é, de certa forma, já esperado nesta faixa etária em que diferenças de gênero não são tão evidentes e a maior parte das intoxicações ocorre por exposições dentro do domicílio e são, em geral, acidentais. ${ }^{4-6,17,18}$ Em relação à idade, notou-se uma variação significante com as intoxicações por medicamentos aumentando com a idade enquanto decresceu a participação dos produtos químicos de uso doméstico. Não existem maiores evidências de que a redução de $8 \%$ no número total de casos anuais de intoxicações, reflexo da diminuição em $1 / 4$ das intoxicações por produtos químicos de uso doméstico, possa ser atribuída a qualquer tipo de ação de saúde pública especificamente orientada para tal problema no período estudado. É possível que se trate apenas de flutuação aleatória ou erros no diagnóstico, registro ou na extração dos dados. Entretanto, seria interessante um monitoramento deste fenômeno por mais alguns períodos para averiguar se este comportamento é sustentado, o que poderia refletir o impacto de recomendações gerais de associações médicas e profissionais de saúde sobre a necessidade de maior cuidado no armazenamento de produtos domésticos.

Os resultados deste estudo devem ser recebidos com cautela. Trata-se de um estudo retrospectivo, baseado em dados registrados em boletins de atendimento de emergência e que cobre apenas uma parcela da população que procura serviços de emergência no Rio de Janeiro. Além das reconhecidas limitações de estudos deste tipo, em particular a baixa qualidade ou mesmo ausência de informações relevantes registradas, há também de se considerar a especificidade da população estudada, que representa somente a parcela das intoxicações infantis que procura e dá entrada em serviços públicos de urgência e que está inserida em um contexto sóciocultural distinto tanto de outras regiões do país, quanto mais de outros países. De qualquer forma, dado a relativa ausência de informações sobre o assunto em nosso meio, este trabalho pode trazer elementos que contribuam para aumentar nossa compreensão sobre este fenômeno.

\section{Conclusão}

Finalmente, os resultados aqui apresentados reforçam a importância de se incluir as intoxicações exógenas no elenco de agravos objeto de ações de vigilância em saúde. Neste sentido, tornar a notificação compulsória e fortalecer a rede de CCls poderiam contribuir para um melhor conhecimento da situação deste problema. Adicionalmente, a adoção da Embalagem Especial de Proteção à Criança (EEPC) em medicamentos e produtos químicos de uso doméstico que apresentem potencial de risco à saúde, a maior fiscalização sobre a comercialização e a apreensão do chumbinho, maior ênfase em atividades de informação e educação em saúde são ações fundamentais para mudar este panorama, que apesar de ainda pouco estudado, já se configura em um grave problema de saúde pública em nosso meio.

\section{Suporte Financeiro:}

Agência Nacional de Vigilância Sanitária e Bayer CropScience

Conflito de interesse: não há

\section{SUMmaRY}

Profile of hospital admissions dUe to acute poisoning among CHILDREN UNDER 6 YEARS OF AGE IN THE METROPOLITAN REGION OF RIO DE JANEIRo, Brazil

OBJECTIVE. To describe the profile of poisoning cases among children attended during three years at emergency hospitals in the metropolitan area of Rio de Janeiro, Brazil.

Methods. All confirmed or suspected poisoning cases, due to drugs and biological substances; organic solvents and hydrocarbons; chemical products; carbon monoxide and other gases; and pesticides, were collected.

RESULTS. 1,574 cases of poisoning in children up to 5 years of age were detected. Around $40 \%$ of the cases involved chemical products of domestic use, 35\% were caused by drugs, and 15\% by pesticides. More than half of pesticide poisonings involved the "chumbinho", an illegal product sold as a rodenticide, and usually including in its formulation a carbamate. Distribution of agents varied significantly by gender, the relative frequency of 
poisonings due to drugs and "chumbinho" being higher among females than males. Participation of chemical products of domestic use decreased with age, but the role of drugs increased as agents of poisoning. During the observation period there was no significant reduction in the total number of annual cases.

CONCLUSION. Supporting the expansion of the network of Poison Control Centers, developing intervention for the production of safer packaging devices for drugs chemical products, suppressing the illegal commerce of "chumbinho", and fostering health education activities might contribute to modify this situation. [Rev Assoc Med Bras 2009; 55(3): 302-7]

KEY WORDS: Toxicology, Poisoning, Pesticides, Drugs. Accidents Pediatrics.

\section{REFERÊNCIAS}

1. WHO. World Health Organization. World Health Report 2004: changing history. Geneve: World Health Organization; 2004.

2. Warner M, Barnes PM, Fingerhut LA. Injury and poisoning episodes and conditions: National Health Interview Survey, 1997. Vital Health Stat. 2000;10(202).

3. Goepp JGK. Pediatric poisonings. Clin Chem. 1996;42:1356-60.

4. Marchi AG, Renier S, Messi G, Barbone F. Childhood poisoning: a population study in Trieste, Italy, 1975-1994. J Clin Epidemiol. 1998;51:687-95.

5. Hoy JI, Day Lm, Tibballs J, Ozanne-Smith J. Unintentional poisoning hospitalisations among young children in Victoria. Inj Prev. 1999;5:31-5.

6. Ramos CLJ, Targa MBM, Stein AT. Perfil das intoxicações na infância atendidas pelo Centro de Informação Toxicológica do Rio Grande do Sul (CIT/RS), Brasil. Cad Saude Publica. 2005;21:1134-41.

7. Margonato FB, Thomson Z, Paoliello MMB. Determinantes nas intoxicações medicamentosas agudas na zona urbana de um município do Sul do Brasil. Cad Saude Publica. 2008;24:333-41.
8. Watson WA, Litovitz TL, Rodgers GC Jr, Klein-Schwartz W, Reid N, Youniss J, et al. 2004 Annual report of the American Association of Poison Control Centers Toxic Exposure Surveillance System. Am J Emerg Med. 2005; 23:589-666.

9. SINITOX. Sistema Nacional de Informações Tóxico-Farmacológicas. [citado 15 fev 2006]. Disponível em: http://www.fiocruz.br/sinitox/2003/apresent2003. htm.

10. Reith DM, Pitt WR, Hockey R. Childhood poisoning in Queensland: An analysis of presentation and admission rates. J Paediatr Child Health. 2001;37:446-50.

11. Ozanne-Smith J, Day L, Parsons B, Tibbals J, Dobbin M. Childhood poisoning: access and prevention. J Paediatr Child Health. 2001;37:262-5.

12. Bochner R. Papel da Vigilância Sanitária na prevenção de intoxicações na infância. REVISA. 2005;1:50-7.

13. Brasil. Congresso Nacional. Câmara dos Deputados. Projeto de Lei n.4841, de 1994, de Fábio Feldman. Determina a utilização de embalagem Especial de Proteção à Criança - EEPC - em medicamentos e produtos químicos de uso doméstico que apresentem potencial de risco à saúde. Brasília (DF): Congresso Nacional. [citado 21 out 2006]. Disponível em: http://www2.camara.gov.br/ proposicoes.

14. Corrêa CL, Zambrone FAD, Cazarin KCC. Intoxicação por "chumbinho": um desafio para o diagnóstico clínico e para o tratamento. Revista Brasileira de Toxicologia 2004; 17: 71-78.

15. Fathelrahman AI, Ab-Rahman AF, Zain ZM. Demographic features of drug and chemical poisoning in northern Malaysia. Clin Toxicol. 2005;43:89-94.

16. Ozdogan H, Davutoglu M, Bosnak M, Tutanc M, Haspolat K. Pediatric poisonings in southeast of Turkey: epidemiological and clinical aspects. Hum Exp Toxicol 2008;27:45-8.

17. Lam LT. Childhood and adolescence poisoning in NSW, Australia: an analysis of age, sex, geographic, and poison types. Inj Prev 2003;9:338-42.

18. Mert E, Bilgin NG. Demographical, aetiological and clinical characteristics of poisonings in Mersin, Turkey. Hum Exp Toxicol 2006;25:217-23.

Artigo recebido: 09/01/08 Aceito para publicação: 11/12/08 